\title{
The Optical and Near-Infrared Morphologies of Isolated Early Type Galaxies
}

\author{
James W. Colbert ${ }^{1}$, John S. Mulchaey ${ }^{2}$, Ann I. Zabludoff ${ }^{3}$
}

\begin{abstract}
In order to study early type galaxies in their simplest environments, we have constructed a well-defined sample of 30 isolated galaxies. The sample contains all RC3 early-type galaxies with no other cataloged galaxy with known redshift lying within a projected radius of $1 \mathrm{~h}_{100}^{-1} \mathrm{Mpc}$ and $\pm 1000 \mathrm{~km} \mathrm{~s}^{-1}$ (where we use the recessional velocities in the RC3). We have obtained optical and near-infrared images of 23 of the galaxies and of a comparison sample of 13 early-type galaxies in X-ray detected poor groups of galaxies. We have applied the techniques of unsharp masking, galaxy model division, and color maps to search for morphological features that might provide clues to the evolution of these galaxies. Evidence for dust features is found in approximately $75 \%$ of both the isolated and group galaxies (17 of 22 and 9 of 12, respectively). However, shells or tidal features are much more prevalent in our isolated sample than in our group sample ( 9 of $22=41 \%$ versus 1 of $12=8 \%$, respectively). The isolation and colors of these shell galaxies make it unlikely that tidal interactions or asymmetric star formation are the causes of such features. One model that is not ruled out is that mergers produce the shells. If shells and dust are both merger signatures, the absence of shells in group ellipticals implies that shells: 1) form more easily, 2) are younger, and/or 3) are longer-lived in isolated environments.
\end{abstract}

Subject headings: galaxies: evolution — galaxies: elliptical and lenticular — galaxies: structure

\section{Introduction}

In the coarsest description of the Hubble sequence, early type galaxies (ellipticals, S0's) are characterized by featureless light profiles, whereas the disks of spiral galaxies have distinct structures like dust lanes and arms. Nevertheless, there is evidence for structure in some early-types. Shells (or ripples) are detected in at least 10\% of the ellipticals in the sample of Malin \& Carter (1983).

\footnotetext{
${ }^{1}$ Department of Astronomy, UCLA, Los Angeles, CA 90095, e-mail: colbert@astro.ucla.edu

${ }^{2}$ Observatories of the Carnegie Institution of Washington, 813 Santa Barbara St., Pasadena, CA 91101, e-mail: mulchaey@ociw.edu

${ }^{3}$ Astronomy Department and Steward Observatory, University of Arizona, Tucson, AZ 85721, e-mail: azabludoff@as.arizona.edu
} 
Large dust lanes or patches are observed in many early-type galaxies (Ebneter et. al. 1988) and are suggested in others by IRAS detections at 60 and $100 \mu \mathrm{m}$ (Thronson \& Bally 1987; Marston 1988; Thronson et al. 1989; Knapp et al. 1989; Bregman et al. 1998). One problem in determining the incidence and causes of such features is accounting first for the role of environment. Most early types lie in clusters and rich groups of galaxies - hot, dense regions that may affect both the formation and evolution of features. It is therefore essential to identify a control sample in truly isolated environments, where the local galaxy density is lower than in even poor groups of galaxies.

We have compiled a sample of 30 isolated early type galaxies in the nearby universe. If we define isolation as having no massive companion within $1 h^{-1} \mathrm{Mpc}$, then no close passage with another galaxy at a relative velocity of $\sim 300 \mathrm{~km} \mathrm{~s}^{-1}$ (approximately the mean pair-wise velocity of galaxies; Marzke et al. 1995) could have occurred in the last $\sim 3 h^{-1}$ Gyr (unless it resulted in a merger). To detect features such as dust and shells, it is necessary to apply a range of image processing techniques to optical and near-infrared images, identifying which methods work best for each type of structure. In particular, we would like to know 1) the fraction of dusty isolated early types, 2) the fraction of isolated early types with shells, and 3) how the fractions of galaxies with dust and with shells/tidal tails vary with galaxy environment.

The fraction of isolated early types that are dusty and/or disturbed offer clues to the evolution of these galaxies. For instance, Malin \& Carter (1983) find that shells or ripples occur roughly five times more frequently in environments outside of rich clusters. Ebneter et al. (1988) argue a possible trend for early-type galaxies outside of clusters to be dustier than their cluster counterparts. These results suggest that, outside of clusters, such features are either easier to form, younger, or longer-lived. However, both the Malin \& Carter (1983) and Ebneter et al. (1988) samples contained few isolated galaxies. A large study of truly isolated galaxies is required to properly address the issue of environmental differences in the structure of early types.

The frequency of dusty and morphologically disturbed isolated galaxies can also illuminate the cause of structure in early types. The origins of such features are currently unknown. Shells, for example, could result from a merger of galaxies (Quinn 1984; Hernquist \& Spergel 1992), weak tidal interactions between galaxies (Thomson \& Wright 1990; Thomson 1991), or asymmetric star formation (Lowenstein, Fabian, \& Nulsen 1987). If shells are found in isolated galaxies, the tidal interactions model could likely be ruled out. If the colors of isolated galaxies with shells are similar to those without shells, then the asymmetric star formation model is less compelling. With a welldefined sample of isolated early types, it is possible to test the validity of different shell formation models for the first time in a simple environment.

In this paper, we analyze the sample of 30 isolated early type galaxies and a comparison sample of 13 poor group ellipticals. We define these samples in $\S 2$. We discuss the optical and near-infrared observations and data reduction in $\S 3$ and $\S 4$. We then search these images for structure. To assist in the detection of features, we use several different analysis techniques, including unsharp masking, model division and color mapping. We describe this data analysis in section $\S 5$. We give our 
results and discuss their relevance to the possibility of mergers in section $\S 6$. Finally, we present our conclusions in $\S 7$. All distances and luminosities in this paper are calculated assuming a $\mathrm{H}_{\mathrm{o}}=$ $100 \mathrm{~km} \mathrm{~s}^{-1} \mathrm{Mpc}^{-1}$ and $\mathrm{q}_{\mathrm{o}}=0.5$ cosmology.

\section{Sample Definition}

Using the new availability of large online databases, we have compiled a unique and welldefined sample of 30 isolated early-type galaxies from the RC3 catalog (de Vaucouleurs et. al. 1991). These galaxies have no cataloged galaxies with known redshifts within a projected radius of $1 \mathrm{Mpc} \mathrm{h}_{100}^{-1}$ and $\pm 1000 \mathrm{~km} \mathrm{~s}^{-1}$. We include all revised morphological Hubble types, $\mathrm{T} \leq-3$. We further restrict our sample to galaxies with $\mathrm{z}<0.033$, within which the isolation can be reliably tested. Due to the incompleteness of the RC3 catalog, we also inspect each galaxy in sky survey images and compare them with group and cluster catalogs drawn from magnitude-limited redshift surveys (NASA Extra-galactic Database (NED); CfA Redshift Survey, Huchra 1995). Galaxies that were in known group or clusters as of October 1995 are excluded from our sample. Table 1 lists our final isolated early-type galaxy sample. The most common galaxy name is given in column 1 , followed by the J2000 right ascension and declination in columns 2 and 3, the recessional velocity in column 4 and RC3 Hubble Type in column 5. The final column contains comments including recently (i.e. since October 1995) cataloged galaxies that are within our isolated criterion (i.e., within a radius of $1 \mathrm{Mpc} \mathrm{h}_{100}^{-1}$ and a velocity of $\pm 1000 \mathrm{~km} \mathrm{~s}^{-1}$ ). We note that several of the isolated galaxies occur at very low Galactic latitude. The high level of extinction in these fields likely plays an important role in these objects being defined as "isolated" . Galaxies at very low Galactic latitude are noted in Table 1 in the Comments column.

We also examined a sample of 13 poor group ellipticals for comparison. These groups are all X-ray detected with spectroscopically confirmed memberships, so they are likely to be bound systems and not chance superpositions (cf. Zabludoff \& Mulchaey 1998). The poor group elliptical sample is given in Table 2. Figure 1 is a histogram comparing the absolute B-band magnitudes of our isolated and poor group samples. Based on a K-S test, the absolute magnitude distributions for the two samples are indistinguishable. However, on average, the group galaxies are closer than the isolated galaxies. Therefore, because both samples were observed under similar conditions (see $\S 3$ and $\S 4$ ), the linear resolution of our images is better on average for the group galaxies (Figure 2). In addition, the signal-to-noise is somewhat better for our group sample. Even for the nearest galaxies in our sample, we image each field out to a radius of at least $\sim 100 \mathrm{~h}_{100}^{-1} \mathrm{kpc}$. Because shell and tidal features are expected on scales of tens of kiloparsec, field of view should not limit our ability to detect structure in the nearer objects. Thus, given the improvements in signal-to-noise and resolution, we are probably more sensitive to detecting structure in our group sample than in our isolated sample, a factor that does not affect our conclusions $(\S 7)$. 


\section{Optical Observations and Data Reduction}

Optical images were obtained at Las Campanas and Palomar Observatories. The Las Campanas images were taken with a Johnson B and a Kron-Cousins R filter at the 40 inch telescope in October 1995 and February 1996. The detector was a Tektronix 2048x2048 CCD with fairly flat response between $\mathrm{B}$ and $\mathrm{R}$. The pixel scale was $0.696 \operatorname{arcsec}_{\mathrm{pixel}}{ }^{-1}$, giving a total field of view of $23.8^{\prime} \times 23.8^{\prime}$. All of the images taken at Las Campanas were taken under photometric conditions with the exception of the group elliptical NGC 533. The measured seeing was in the range of $~$ 0.9-1.9 arcsec, except for the galaxy NGC 3209, which was observed at very high airmass. For each galaxy, two 150 second integrations were obtained in each band. A total of 19 isolated galaxies and 11 group ellipticals were observed during the two Las Campanas observing runs.

Three isolated ellipticals and one group elliptical were observed at the Palomar Observatory 60 inch telescope in November 1995. The filters used were the Johnson B and I bands. The conditions were non-photometric during this observing run, so no attempt was made to measure magnitudes for these galaxies. However, the seeing was very good $(\sim 0.8-1.2 \operatorname{arcsec})$.

The optical data were reduced using standard techniques in IRAF. The bias level was determined from the overscan region and then subtracted from the images. Flat-fielding was done with twilight flats, typically 5-10 flats for each filter on each night. The flats were medianed together to improve the signal-to-noise ratio and then normalized. Each image was then divided by the normalized flat field and sky subtracted. We determined the sky level by examining several regions on the CCD free of emission from both stars and galaxies. The two 150 second images of each galaxy and band were combined in such a way as to eliminate cosmic ray strikes. The images taken at Las Campanas were photometrically calibrated using standard star fields in Graham (1982). Total magnitudes were measured with the program SExtractor (Bertin \& Arnouts 1996) using a method similar to that proposed by Kron (1980). A comparison of our derived magnitudes with those in the literature suggests a median external error estimate of 0.15 mag. Figure 3 shows the B-R colors we derive for both samples using the Las Campanas observations. The range of colors are comparable for the two samples, suggesting similar stellar populations.

\section{Near-Infrared Observations and Data Reduction}

We obtained near-infrared images of 9 isolated galaxies and 2 group galaxies on two observing runs at the 100 inch telescope at Las Campanas in September 1995 and April 1996. The detector was a Rockwell NICMOS-3 HgCdTe 256x256 array. The pixel scale was $0.35 \operatorname{arcsec~pixel~}^{-1}$, giving a total field of view of approximately $1.5 \times 1.5$ arcminutes. The filter used has a central wavelength around 2.15 microns and a FWHM of 0.32 microns (this band is often referred to as $\mathrm{K}_{\text {short }}\left(\mathrm{K}_{\mathrm{S}}\right)$ ). The conditions during both observing runs were photometric. Seeing varied between 0.6 and 1.2 arcsec, averaging around 0.8-0.9 arcsec. For each galaxy, we adopted an observing procedure identical to that described in Mulchaey et al. (1997). To minimize systematic errors we took 
the galaxy images in a dithered pattern, with 6 twenty second images for each position. An equal amount of time was spent on dithered sky exposures, typically $3-4$ arcminutes from the galaxy. Generally, 13 on-source positions were obtained resulting in a total integration time of approximately 26 minutes. In addition, we also obtained a frame far off to the side of any bright galaxies in order to better estimate the sky level.

The data were reduced following the techniques described in Mulchaey et al. (1997). Flat field images were created for each night using all the sky exposures obtained that night. A sky frame was formed for each pair of object exposures by median filtering the sets of sky exposures taken before and after each object pair. We then applied the flat field to the object frames and subtracted the appropriate sky frame. After mosaicing the six images at each dithered position, we mosaiced the images from all thirteen dithered positions together. In most cases, stars were used to register the images. However, in a few cases, the galaxy center was used. Because the galaxy emission clearly extends beyond the edges of our final near-infrared images, we have not tabulated total $\mathrm{K}_{\mathrm{S}}$ magnitudes.

\section{Data Analysis}

The search for dust and tidal features in elliptical galaxies has been greatly enhanced by the development of several data analysis techniques. Here, we use three of the most powerful techniques for revealing structure in elliptical galaxies: unsharp masking, galaxy model division and color mapping. For images taken at Palomar, we substitute the I band image for the R band throughout the following discussion.

\subsection{Unsharp Masking}

Searching for shells, ripples, tidal tails and other faint features in the outer regions of ellipticals is severely hampered by the strong radial intensity gradient of the underlying galaxy, which can wash out low surface brightness features. One way of removing this unwanted background light is unsharp masking. This procedure involves taking the original image of the galaxy, smoothing it until all small scale features are erased, and then subtracting this "unsharp" picture from the original galaxy image. Only small scale features, such as thin shells or tails, then remain. For smoothing we follow the method of McGaugh \& Bothun (1990) and use a median filter. This method does not produce good results for galaxy centers where the brightness gradient is strong, but is excellent for finding details at the outer edges. After testing a range of median filter sizes, we found that a filter of 10-20 arcseconds generally worked best at revealing features. For the velocity range of our galaxies (5000-10,000 $\mathrm{km} \mathrm{s}^{-1}$ ), this filter size corresponds to a physical size of $\sim 6 \mathrm{~h}_{100}^{-1}$ kpc. In all cases where a feature is uncovered using the unsharp masking technique, we are able to identify the feature in the original broad-band image by adjusting the stretch of the display. 


\subsection{Galaxy Model Division}

While unsharp masking works well for the outer regions of a galaxy, the inner region is also of interest. Again we have the problem of a severe radial gradient in light intensity. To attack this problem we choose the method of model division, used successfully by Ebneter, Djorgovski \& Davis (1988), to identify dust features in galaxies. Using the ELLIPSE program contained in IRAF's STSDAS package, we fit elliptical isophotes to all galaxies in all available filters. Using these models and the IRAF program BMODEL we then make an artificial copy of the galaxy. To avoid contamination by stars or background galaxies, ELLIPSE is set to reject 10-20\% of pixels farthest from the median surface brightness value as it samples each isophote. While ELLIPSE has difficulty modeling the inner 2-3 pixels, it provides a good model for the galaxy overall. To search for large variations from simple elliptical isophotes, we divide the original galaxy image by the model image and look for residual features. While Ebneter, Djorgovski \& Davis (1988) go through a process of smoothing the outer regions of their models while leaving the centers alone, we skip that step, choosing instead to handle the analysis of the outer regions with the unsharp masking technique.

Several of our ellipticals show an odd "quadrupole" feature near the galaxy center in the model division image. A similar feature was seen by Ebneter et al. (1988). After reprocessing the images with a slightly different choice for the galaxy center, Ebneter et al (1988) found that several of their images no longer showed a quadrupole, but instead revealed a disk. From this result Ebneter et al. (1988) concluded that it is likely that all galaxies with quadrupole features have disks, which make the total isophotes non-elliptical and cause a numerical instability in the isophote fitting algorithm. In cases where the quadrupoles could not be removed, Ebneter et al (1988) concluded that the galaxies contained unresolved disks. However, Lauer (1985) shows that boxiness in the stellar orbits can also cause a quadrupole (in his case, a significant fourth Fourier harmonic of the pixel intensities). Model division of the boxy, poor group elliptical NGC 4261 (Lauer 1985), for example, produces a quadrupole image with a phase that aligns roughly with the diagonal axis of the galaxy's box in the unsharp mask image (see Figure 7). We do not try to discriminate between quadrupoles resulting from boxiness or diskiness in this paper.

Following Ebneter et al. (1988), we reprocess our images by varying the galaxy center used in our ellipse model. This approach is successful in several cases, revealing apparently flattened, dusty structures. In addition to NGC 4261, we are unable to remove quadrupoles from the isolated elliptical NGC 3332 and the poor group elliptical NGC 5129.

In the case of the morphologically disturbed galaxy, IC 2637, we cannot obtain an adequate model of the galaxy isophotes using ELLIPSE. Thus, we do not apply the model division technique to this galaxy. 


\subsection{Color Mapping}

Features in elliptical galaxies can also be revealed by color maps. This technique has been used by many authors, including Ebneter \& Balick (1985) and Regan, Vogel \& Teuben (1995). For galaxies for which we possess data in $\mathrm{B}, \mathrm{R}$, and $\mathrm{K}_{\mathrm{S}}$, we make three color maps: $\mathrm{B}$ divided by $\mathrm{R}, \mathrm{R}$ divided by $\mathrm{K}_{\mathrm{S}}$, and $\mathrm{B}$ divided by $\mathrm{K}_{\mathrm{S}}$. In all images we observe a reddening color gradient toward the center, a phenomenon observed before and thought to be associated with an increasing metallicity toward the center of elliptical galaxies (e.g. Davies, Sadler \& Peletier 1993; Trager et al. 2000). Due to the intensity gradient of the galaxy, proper alignment of the various images is very important. Slight misalignment leads to obvious dipoles in the final color map. We are careful to match the pixel scale and resolution of the images in the three different bands. We observe structure in the color maps of 26 of the 34 galaxies that we observed in multiple bands.

\section{Results and Discussion}

The identification of morphological structures is largely a subjective process. For our purposes here, we define structure as any feature that deviates from simple elliptical isophotes. Each image was examined for features independently by J. Colbert and J. Mulchaey. Only features identified by both of these authors are considered real. There is good agreement between them, both authors identifying 54 separate features while disagreeing on only four. The results of each technique are given in Table 3 for the isolated sample and in Table 4 for the group galaxies. A more detailed description of the features found in each case is given in Appendices A and B, and a few example images are given in Figures $4-7$.

All three of the data analysis techniques that we employ reveal features in our early-type galaxy sample. In general, two types of features are found: 1) shell or tidal features visible near the outskirts of the stellar distribution and 2) dust features. The difference between a "shell" and a tidal feature is ambiguous in these images, and thus we do not make a physical distinction between these two types of features in this paper.

Dust lanes and patches are the most common type of feature we find. While both the model division and color map techniques are successful at uncovering dust, color maps provide the most sensitive probe of dust features. Our color maps reveal dust in approximately $75 \%$ of the galaxies observed, with similar detection rates for the isolated and group samples. The model division technique reveals dust features in $\sim 48 \%$ (10 of 21 ) of the isolated galaxies and $42 \%$ ( 5 of 12 ) of the group galaxies. In addition, one isolated galaxy and two group galaxies display quadrupole features that could be associated either with boxiness or a central disk. Counting these galaxies among those with detected dust brings the percentage of galaxies displaying dust in the model division maps to $\sim 52 \%$ (11 of 21 ) for the isolated sample and $\sim 58 \%$ (7 of 12) for the group sample. All the dust features detected in the model division maps are also detected in the color maps. This result suggests that although the model division method is not the best way to find dust in early-type 
galaxies, the technique is useful, especially if only one band is available. It is also worth noting that the majority of dust patches found are very close to the center of the galaxy (usually within the inner $\mathrm{h}_{100}{ }^{-1} \mathrm{kpc}$ ). However, because the signal to noise ratio tends to be highest near a galaxy's center, our survey is most sensitive to dust there.

To calculate the probability that the dust fractions are the same in the two samples, we use the cumulative binomial probability distribution to find if there is a "parent" dust fraction consistent with both the isolated and group galaxy dust fractions. For example, suppose that $k_{1} / n_{1}$ is the observed fraction of isolated galaxies that have dust and $k_{2} / n_{2}<k_{1} / n_{1}$ is the observed fraction of group galaxies that have dust. For each parent probability $p=0$ to 1 , we calculate the probability $P_{1}$ that at least $k_{1}$ of $n_{1}$ isolated galaxes have dust,

$$
P_{1} \equiv \sum_{j=k_{1}}^{n_{1}}\left(n_{1}\right) p^{j}(1-p)^{n_{1}-j}=I_{p}\left(k_{1}, n_{1}-k_{1}+1\right),
$$

where $I_{p}$ is the incomplete beta function.For the same $p$, we calculate the probability $P_{2}$ that at most $k_{2}$ of $n_{2}$ group galaxies are dusty,

$$
P_{2} \equiv 1-I_{p}\left(k_{2}+1, n_{2}-k_{2}\right) .
$$

The product of the probability distributions $P_{1}(p) P_{2}(p)$ is the joint probability distribution, whose maximum tells us the likelihood that both $k_{1} / n_{1}$ and $k_{2} / n_{2}$ were drawn from the same parent fraction. The probability that the dust fraction of the isolated sample is the same as that of the group sample is 0.3317 (i.e., not statistically different).

All the shell features that we uncover are discovered by applying the unsharp masking technique to our R-band images. While some of these features are also visible in the B-band images, none are detected in the $\mathrm{K}_{\mathrm{S}}$ images. Particularly noteworthy is the galaxy NGC 7010, which is known to contain prominent shells (McGaugh \& Bothun 1990), but shows no evidence for any structure in our $\mathrm{K}_{\mathrm{S}}$ image (the shells are easily visible in our $\mathrm{R}$ and $\mathrm{B}$ images). The failure to detect shells in the $\mathrm{K}_{\mathrm{S}}$ band may be an indication that such features are below the surface brightness limit of the images. For this reason, we exclude from the tidal feature statistics the two galaxies that are only observed in $\mathrm{K}_{\mathrm{S}}$.

We detect shell/tidal features in 9 of the 22 isolated galaxies $(\sim 41 \%)$. (The percentage is roughly the same for galaxies classified as ellipticals and lenticulars.) However, only one of the 12 group early-type galaxies shows evidence for shells $(\sim 8 \%)$. We should be more sensitive to the presence of shells in the group sample given that this sample is closer on average than the isolated sample (see §2). Using the binomial probability distribution discussed above, the probability that the fraction of galaxies with shell/tidal features is the same for both samples is only 0.016. The tendency for shells to be found preferentially around ellipticals in low density environments was first suggested by Malin \& Carter (1983), who noted that most shell galaxies are either apparently isolated or in very poor groups. As the groups in our sample are all X-ray detected, they are likely considerably richer than the typical group in Malin \& Carter (1983). On the other hand, our 
isolation criteria ensure that the isolated sample galaxies lie in more rarefied environments than is typical for the shell galaxies in Malin \& Carter (1983).

Our image analysis demonstrates that isolated elliptical galaxies are not featureless: $\sim 75 \%$ of our isolated sample have dust features and $41 \%$ show evidence for shell/tidal features. Because the factors that may affect the evolution of early types in isolated environments are different than those in clusters and poor groups, the isolated sample is a useful control when compared to denser environments. The fraction of dusty galaxies is similar for the group and isolated samples. The shell discovery rate, on the other hand, is clearly dependent on environment, being significantly lower in the poor group sample.

For the isolated galaxies, tidal interactions with other galaxies (Thomson \& Wright 1990; Thomson 1991) are an unlikely mechanism for shell formation as such features are not expected to last more than $\sim 1 \mathrm{~h}_{100}^{-1}$ Gyr (Quinn 1984) and our isolation criteria exclude encounters within the last $\sim 3 \mathrm{~h}_{100}^{-1}$ Gyr. The consistancy of the B-R colors of the isolated galaxies with and without shells ( $\mathrm{B}-\mathrm{R}=1.45$ vs $\mathrm{B}-\mathrm{R}=1.44$, galaxies with $\mathrm{A}_{\mathrm{B}} \leq 0.3$ only) argues against the picture in which shells are caused by asymmetric star formation (Lowenstein et al. 1987). However, galaxy-galaxy mergers are still a viable option. If mergers are responsible for introducing dust into early-type galaxies (i.e. Morganti et al. 1997), than the similar dust fractions in our isolated and group samples suggest mergers have been important in both environments.

If shells and tidal features are also an indication of mergers, then the differences between the isolated and group shell fractions means we need to explain the differences between the merger histories of galaxies in these two different environments. One possibility is that dust features are produced by a wide range of galaxy-galaxy mergers, while shells form in more limited types of mergers that do not occur in denser environments. For example, shells may form when a gas rich dwarf is swallowed by a larger galaxy (Weil \& Hernquist 1993). However, gas rich dwarfs may not exist near the centers of X-ray detected groups, where the intragroup medium and galaxy number densities are highest. A second possibility is that even if shells do form in X-ray group galaxies, the lifetime of such features is reduced by the hotter, denser environment. A third possibility is that because extended tidal/shell features are expected to only have a limited lifetime $\left(\sim 10^{9}\right.$ years, Quinn 1984), the difference in the isolated and group environments simply reflects that the isolated ellipticals have interacted more recently. However, the mean colors (B-R) of the isolated and group samples are similar (see Figure 3), so if interactions have occurred more recently in the isolated ellipticals, there has been very little if any effect on the stellar populations of the galaxies.

It is also interesting to note that all of the galaxies that display shell/tidal features also contain dust. Given the dust and shell detection rates for the isolated sample (77\% and $41 \%$, respectively), we would expect $\sim 32 \%$ (i.e., $0.77 \times 0.41$ ) or 7 of the 22 isolated galaxies to display both dust and shells. The actual number of isolated galaxies with observable dust and shells is 9 , which is not different from 7 at a statistically significant level. Thus, we are unable at the present time to determine if dust and shells are correlated for isolated early-type galaxies. 


\section{Conclusions}

We use multi-color images of a sample of isolated, early type galaxies to study the properties of such galaxies outside the hot, dense environments of rich clusters. We compare this sample to a sample of early types in X-ray detected poor groups. Our survey has the important advantage over previous studies that our strict isolation criteria ensures that no recent galaxy or environment interaction is possible except for a complete merger.

The majority of galaxies in our sample show evidence for dust $(\sim 75 \% ; 17$ of 22 for the isolated sample and 9 of 12 for the group sample). We apply two different techniques to search for dust features, model division and color maps. Both methods suggest that the incidence of dust is comparable in isolated and poor group ellipticals. However, the color maps are more successful at uncovering dust (approximately one-third of the galaxies that show evidence for dust in the color maps show no evidence for dust in the model division images). The dust detection rates are identical for our B-K $\mathrm{K}_{\mathrm{S}}$ and B-R images. Thus, although dust features tend to be most prominent in optical/near-infrared color maps, the optical images alone appear adequate for identifying dust features.

We have used the technique of unsharp masking to search for shells and tidal features. We found these features in 41\% (9 of 22) of our isolated galaxies and approximately $8 \%$ ( 1 of 12 ) of our group galaxies. The isolation and colors of the shell galaxies suggest that neither tidal interactions nor asymmetric star formation cause such features. A more likely model is that mergers produce the shells. Although the dust fraction is virtually identical for the isolated and group environments, the shell fractions are significantly different. If shells and dust are both merger signatures, the absence of shells in group ellipticals implies that shells: 1) form more easily, 2) are younger, and/or 3) are longer-lived in more isolated environments.

We thank Chuck Keeton for providing useful information on statistics and the anonymous referee for comments that improved this paper. We would also like to thank Tod Lauer for his

helpful suggestions. This research involved the use of the NED database. Partial support for this program was provided from NASA grants NAG 5-3529 and HF-01087.01-96A. 


\section{A. Comments On Individual Galaxies - Isolated Galaxies}

NGC 179- The B/R color map reveals large dust patches around the center of the galaxy. IRAS detected this galaxy at 60 and $100 \mu \mathrm{m}$ (Knapp et al. 1989).

NGC 766-The B/R image of this galaxy reveals an unusually red core, but does not appear to contain any obvious dust patches. It is also a weak radio galaxy.

NGC 1132-No features are found in this galaxy. ASCA observations indicate that this galaxy is surrounded by a large group-like X-ray emitting halo (Mulchaey \& Zabludoff 1999).

A $0300+16-$ No features are found in this galaxy, which has previously been identified as a FR I radio galaxy (Smith \& Heckman 1989).

UGC 02748-The B/R color map reveals several small dust patches, including a strong one near the center. This galaxy is a known FR II radio galaxy.

A $0356+10-$ No features are detected in this FR II radio galaxy.

II $\mathrm{Zw}$ 017-The $\mathrm{R} / \mathrm{K}_{\mathrm{S}}$ color map reveals an asymmetric, non-elliptical red feature running through the center, which is probably a dust lane.

NGC 2110-The B/R image reveals dramatic dust lanes near the galaxy's center. These dust lanes have previously been seen in HST images (Mulchaey et al. 1994). IRAS detected this Seyfert 2 galaxy at $12,25,60$ and $100 \mu \mathrm{m}$.

A 0718-34- Unsharp masking reveals a shell/tidal feature to the southeast. The B/R maps show several clear dust patches in the center. This galaxy is a double radio source with several unresolved components (Jones \& McAdam 1992).

NGC 3209- Our images for this galaxy were taken under very poor seeing conditions $\left(\sim 3^{\prime \prime}\right)$. Thus, although we found no evidence for features in this weak radio galaxy, our limits on structure are not very strong.

NGC 3332-This galaxy is clearly disturbed in our R image with a large arm-like feature extending north of the galaxy. It is probably this feature that has led to this galaxy's classification as an S0. While we can not rule out the possibility that this feature is an arm, in the unsharp masked image it looks very sharp and thus more like a shell. The $\mathrm{R}$ model divided image shows a quadrupole feature. The $\mathrm{R} / \mathrm{K}_{\mathrm{S}}$ color map reveals red patches near the galaxy center which are consistent with dust. This galaxy was detected by IRAS at 60 and $100 \mu \mathrm{m}$ (Knapp et al. 1989).

IC 2637 (MRK 732)-IC 2637 is the most morphologically disturbed galaxy we observed. In R band it looks more irregular than elliptical. In the $\mathrm{K}_{\mathrm{S}}$ band at low stretches, a disk or ring structure is evident. At more saturated stretches, the infrared image does resemble an elliptical. All color

maps show a strong dust lane running across the galaxy. There are also several smaller red patches that may be associated with dust. This dust appears to be the major reason for the galaxy's 
irregular appearance in the optical bands. The unsharp mask reveals structure on the north side of the galaxy that may be shells or tidal tails. To the south is a large arc, far enough away to avoid any confusion with the complicated structure of the rest of the galaxy. The morphology of IC 2637 is so distorted that we could not make an adequate model of the galaxy isophotes using the task IRAF Ellipse. Thus, we did not apply the model division technique for this object. This Seyfert 1.5 galaxy (Hewitt \& Burbidge 1991) was detected by IRAS at 12, 60 and $100 \mu \mathrm{m}$ (Moshir et al. 1990).

IC 2980- Color maps reveal dust patches around the nucleus.

ESO 505- G 015- Unsharp masking reveals shells to the southeast and northwest. The B/K map indicates a possible dust patch north of the nucleus.

ESO 065-01- Unsharp masking reveals at least one shell off the major axis of the elliptical. Unfortunately the other side of the elliptical has a bad CCD column nearby, obscuring our ability to see structure on that side. The $\mathrm{R} / \mathrm{K}_{\mathrm{S}}$ color map reveals two red patches situated very assymetrically. The $\mathrm{K}_{\mathrm{S}}$ model divided image does not show any clear structure or bright spots associated with these patches. Dust is therefore a good candidate. In the radio this is a complex source with a strong core and edge darkened lobes(Jones \& McAdam 1992).

ESO 574- G 017-This galaxy is a clearly disturbed, with faint structure extending from it in a bow shock shape. The unsharp masking reveals significant structure. On the east side the structure is close to the galaxy and looks shell-like, whereas on the west side the features are extending away from the galaxy like a tidal tail. The color map reveals several red patches near the center of the galaxy, which are likely due to dust. IRAS detected this galaxy at 25, 60 and $100 \mu \mathrm{m}$ (Thronson et al. 1989).

IC 1156-This galaxy is only observed in the $\mathrm{K}_{\mathrm{S}}$ band, so we did not apply our three analysis techniques to this object.

NGC 6172-Unsharp masking reveals what appears to be a shell to the southeast of NGC 6172, but not very far from its center $\left(\sim 25\right.$ arcsec or $\left.9 \mathrm{~h}_{100}^{-1} \mathrm{kpc}\right)$. The color maps reveal dust near the galaxy center. This galaxy was detected by IRAS at 25 and $60 \mu \mathrm{m}$ (Moshir et al. 1990).

NGC 6799-The color maps reveal a large dust lane along the eastern edge of the galaxy.

NGC 7010-This galaxy has prominent shells that have been reported previously by McGaugh \& Bothun 1990. IRAS detected NGC 7010 at 12 and $100 \mu \mathrm{m}$ (Moshir et al. 1990).

IC 1392-Unsharp masking suggests a shell to the southwest. The B/I image shows a small patch of dust at the galaxy's center.

IC 5258-There is evidence for dust near the center of the B/I image.

NGC 7618- The B/I color image reveals a strong dark dust lane running down the center. 


\section{B. Comments on Individual Galaxies - Poor Group Ellipticals}

NGC 383-A linear dust feature is seen in the color map. IRAS detected this galaxy at 60 and $100 \mu \mathrm{m}$ (Knapp et al. 1989).

NGC 533-We detect no features in this galaxy.

NGC 2563-Forbes \& Thomson (1992) have detected possible shells in this galaxy, but no such feature is found in our somewhat shallower image. The color map reveals a large dust patch near the center of the galaxy.

NGC 3091-This is the most luminous galaxy in HCG 42. No dust or tidal features are detected.

NGC 3557-The color map reveals a possible ring of dust near the center of the galaxy. NGC 3557 is a double tailed radio source with a central knot and a jet (Birkinshaw \& Davies 1985). IRAS detected this galaxy at 12, 60 and $100 \mu \mathrm{m}$ (Knapp et al. 1989).

NGC 4261-The color map shows two large dust patches on either side of what appears to be a bluer central lane. A nuclear disk with a similar orientation, but on a smaller scale $\left(\sim 2^{\prime \prime}\right.$ or 200 $\mathrm{h}_{100}{ }^{-1}$ pc versus our $\sim 8-10^{\prime \prime}$ or $1 \mathrm{~h}_{100}{ }^{-1} \mathrm{kpc}$ structure) has been reported by Jaffe et al. (1996) based on HST images. Because the phase of the quadrupole in the model division image aligns roughly with the diagonal axis of the galaxy's box in the unsharp mask image, the quadrupole is likely to result from the galaxy's boxiness (our Figure 7; Lauer 1985). This galaxy was detected by IRAS at 12, 25, 60 and $100 \mu \mathrm{m}$ (Knapp et al. 1989).

NGC 4325-The color map reveals dust near the galaxy center.

NGC 4759-The second brightest galaxy in HCG 62. The color map reveals strong dust patches near its center.

NGC 4761-The brightest galaxy in HCG 62. We find no evidence for dust or tidal features, although the galaxy is part of an on-going interaction with NGC 4759 (Hickson et al. 1992).

NGC 5044-The color map shows two large dust patches near the galaxy's center, with a smaller patch off to the east. IRAS detected this galaxy at 12, 60 and $100 \mu \mathrm{m}$ (Knapp et al. 1989).

NGC 5129-The model divided image of NGC 5129 has a strong quadrupole. The color map reveals a bluer central lane surrounded by redder dust patches. A possible tidal feature is detected near the southeastern corner of the galaxy.

IC 4296-The color map reveals large dust patches around the center and some indication of a disk. IC 4296, a FR I radio galaxy, was detected by IRAS at 60 and $100 \mu \mathrm{m}$ (Knapp et al. 1989).

NGC 5846-While previous studies suggest NGC 5846 contains dust (Goudfrooij \& Trinchieri 1998), our analysis is incomplete because we only obtained a $\mathrm{K}_{\mathrm{S}}$ image. 


\section{REFERENCES}

Bertin, E. \& Arnouts, S. 1996, å, 117, 393

Birkinshaw, M. \& Davies, R.L. 1985, ApJ, 291, 32-44

Bregman, J. N., Snider, B. A., Grego, L., Cox, C. V. 1998, ApJ, 499, 670

Davies, R.L., Sadler, E.M., \& Peletier, R.F. 1993, MNRAS, 262, 650

de Vaucouleurs, G., de Vaucouleurs, A., Corwin, H.G., Buta, R.J., Patrel, G. \& Foque, P. 1991, "Third Reference Catalogue of Bright Galaxies" (Springer-Verlag: New York)

Ebneter, K. \& Balick, B. 1985, AJ, 90, 183

Ebneter, K., Djorgovski, S., \& Davis, M. 1988, AJ, 95, 422-437

Forbes, D.A. \& Thomson, R.C. 1992, MNRAS, 254, 723

Goudfrooij, P., \& Trinchieri, G. 1998, AA, 330, 123

Graham, J.A. 1982, PASP, 94, 244

Hernquist, L., \& Spergel, D. L., 1992, ApJ, 399, L117.

Hewitt \& Burbidge 1991, ApJS, 75, 297

Hickson, P., Mendes de Oliveira, C., Huchra, J. P., Palumbo, G. G., 1992, ApJ, 399, 353

Huchra, J. 1995, private communication

Jaffe, W., Ford, H., Ferrarese, L., van den Bosch, F. \& O'Connell, R.W. 1996, ApJ, 460, 214

Jones, P.A. \& McAdam, B. 1992, ApJS, 80, 137

Knapp, G.R., Guhathakurta, Kim, D.W. \& Jura, M. 1989, ApJS, 70, 329

Kormendy, J. \& Stauffer, J. 1986, "Dust in Early Type Galaxies Observed at the CFHT", I.A.U. Symp. 127, ed. T. De Zeeuw (D. Reidel Publishing: Dordrecht), p. 405-6.

Kron, R. 1980, ApJS, 43, 305

Lauer, T.R. 1985, MNRAS, 216, 429-438

Loewenstein, M., Fabian, A.C., \& Nulsen, P.E.J. 1987, MNRAS, 229, 129

Malin, D.F. \& Carter, D. 1983, ApJ, 274, 534

Marston, A. P. 1988, MNRAS, 231, 333.

Marzke, R.O., Geller, M.J., da Costa, L.N., Huchra, J.P., AJ, 110, 477

McGaugh, S.S. \& Bothun, G.D. 1990, AJ, 100, 1073

Morganti, R., Sadler, E.M., Oosterloo,T., Pizzella, A., \& Bertola, F. 1997, AJ, 113, 937

Moshir, M., Kopan, G., Conrow, T., McCallon, H., Hacking, P., Gregorich, D., Rohrbach, G., Melnyk, M., Rice, W., Fullmer, L., et al. 1990, Infrared Astronomical Satellite Catalogs, The Faint Source Catalog, Version 2.0 
Mulchaey, J.S., Regan, M.W., Kundu, A. 1997, ApJS, 110, 299

Mulchaey, J.S., Wilson, A.S., Bower, G.A., Heckman, T.M., Krolik, J.H., \& Miley, G. K. 1994, ApJ, 433, 625

Mulchaey, J.S. \& Zabludoff, A. I. 1999, ApJ, 514, 133

Quinn, P.J. 1984, ApJ, 279, 596

Regan, M.W., Vogel, S.N. \& Teuben, P.J. 1995, ApJ, 449, 576

Smith, E.P. \& Heckman, T.M. 1989, ApJS, 69, 365

Thronson, H.A., Bally, J. 1987, ApJ, 319, L63.

Thronson, H.A., Bally, J. \& Hacking, P. 1989, AJ, 97, 363

Thomson, R.C. 1991, MNRAS, 253, 256.

Thomson, R.C. \& Wright, A.E. 1990, MNRAS, 247, 122

Trager, S.C., Faber, S.M., Worthey G., \& Gonzalez, J.J. 2000, ApJ, 119, 1645

Weil, M.L. \& Hernquist, L. 1993, ApJ, 405, 142

Zabludoff, A.I. \& Mulchaey, J.S. 1998, ApJ, 496, 39 


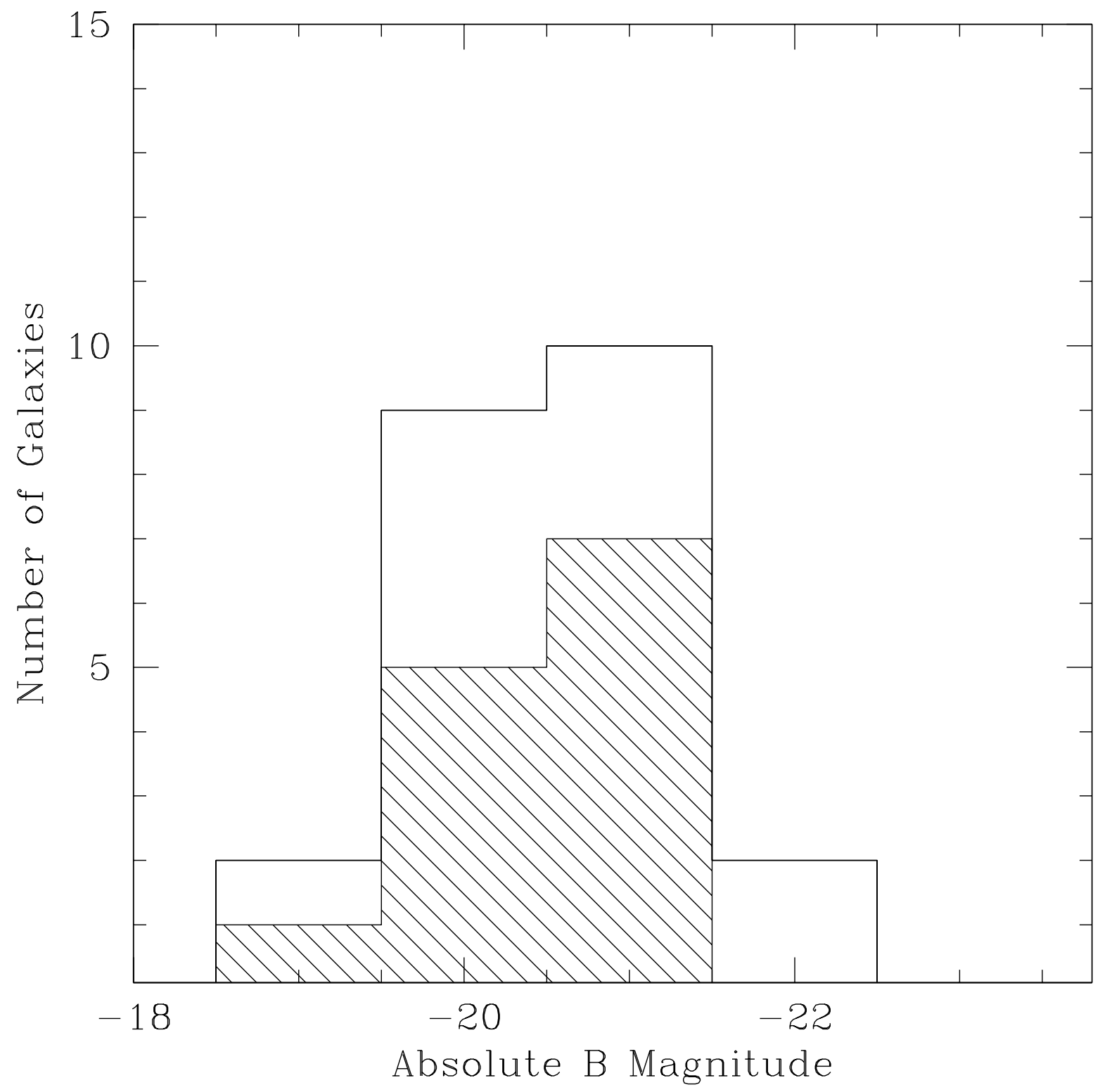

Fig. 1. - Histogram of absolute B magnitude for the isolated early-type sample (open histogram) and the early-type galaxies in poor groups sample (dashed histogram). A K-S test cannot distinguish between the two distributions. 


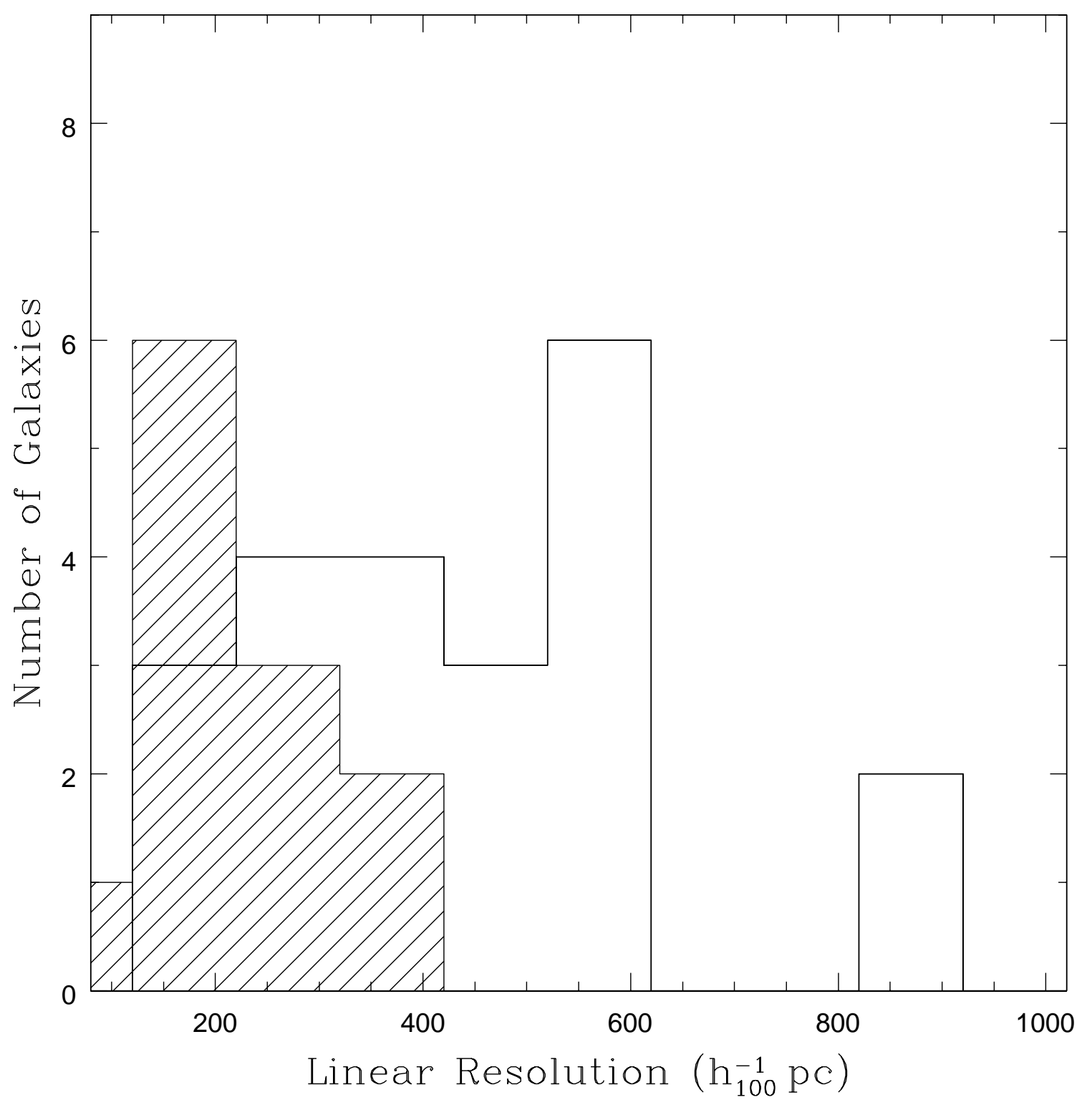

Fig. 2.- Histogram of the linear resolution in $\mathrm{h}^{-1}{ }_{100}$ parsecs for the isolated early-type sample (open histogram) and the early-type galaxies in poor groups sample (dashed histogram). The resolution is based on estimates of the seeing determined using the red image (either $\mathrm{R}$ or I band). A K-S test indicates that the two distributions are different at a significance of greater than $99 \%$. 


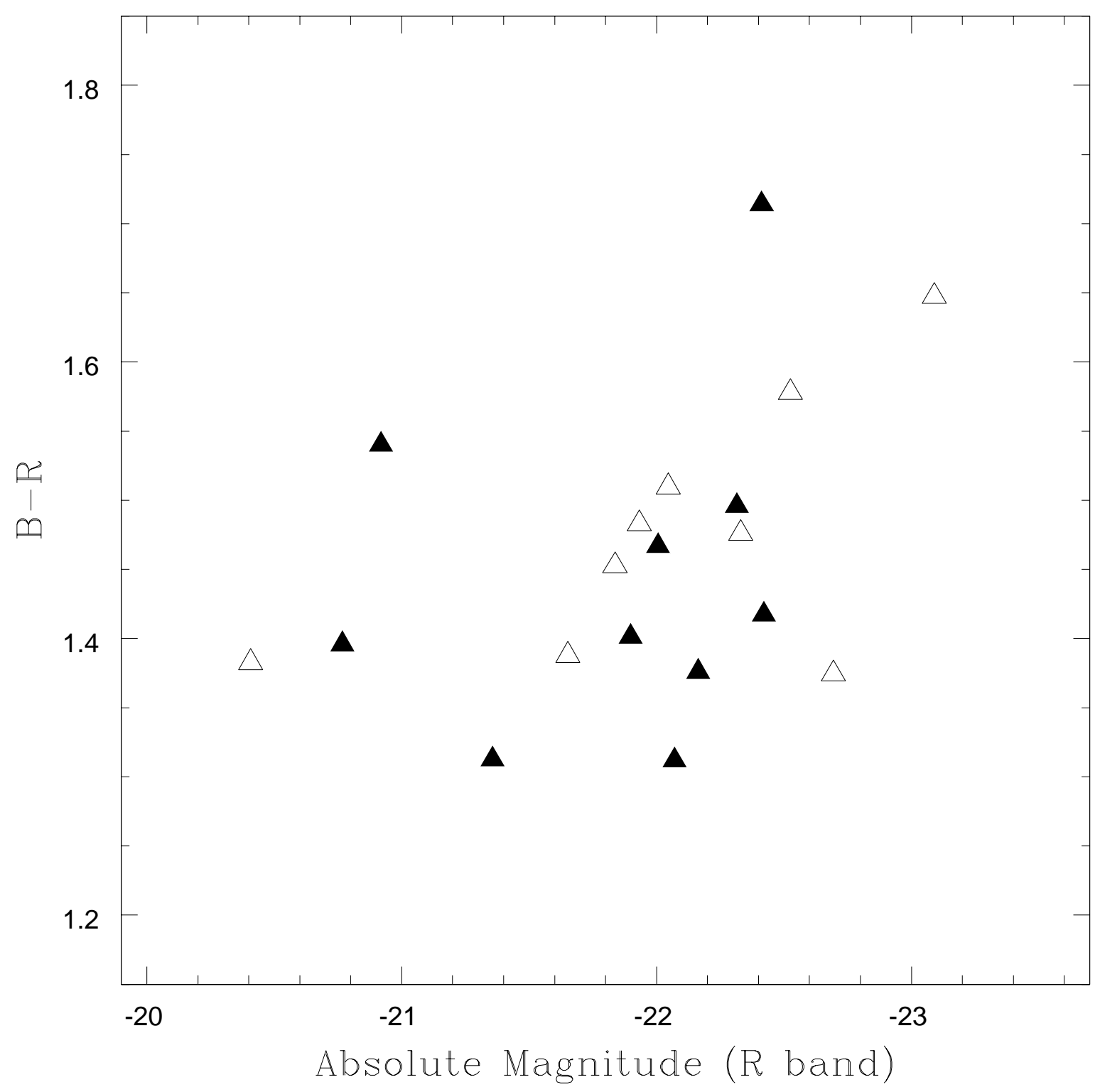

Fig. 3.- Absolute R magnitude vs. B-R color diagram for the isolated early-type sample (open triangles) and the group early-type sample (filled triangles). Only galaxies with Galactic extinction values less than $A_{B}=0.3$ are included. The colors of the galaxies in the two different environments are comparable, suggesting similar stellar populations in these samples. 

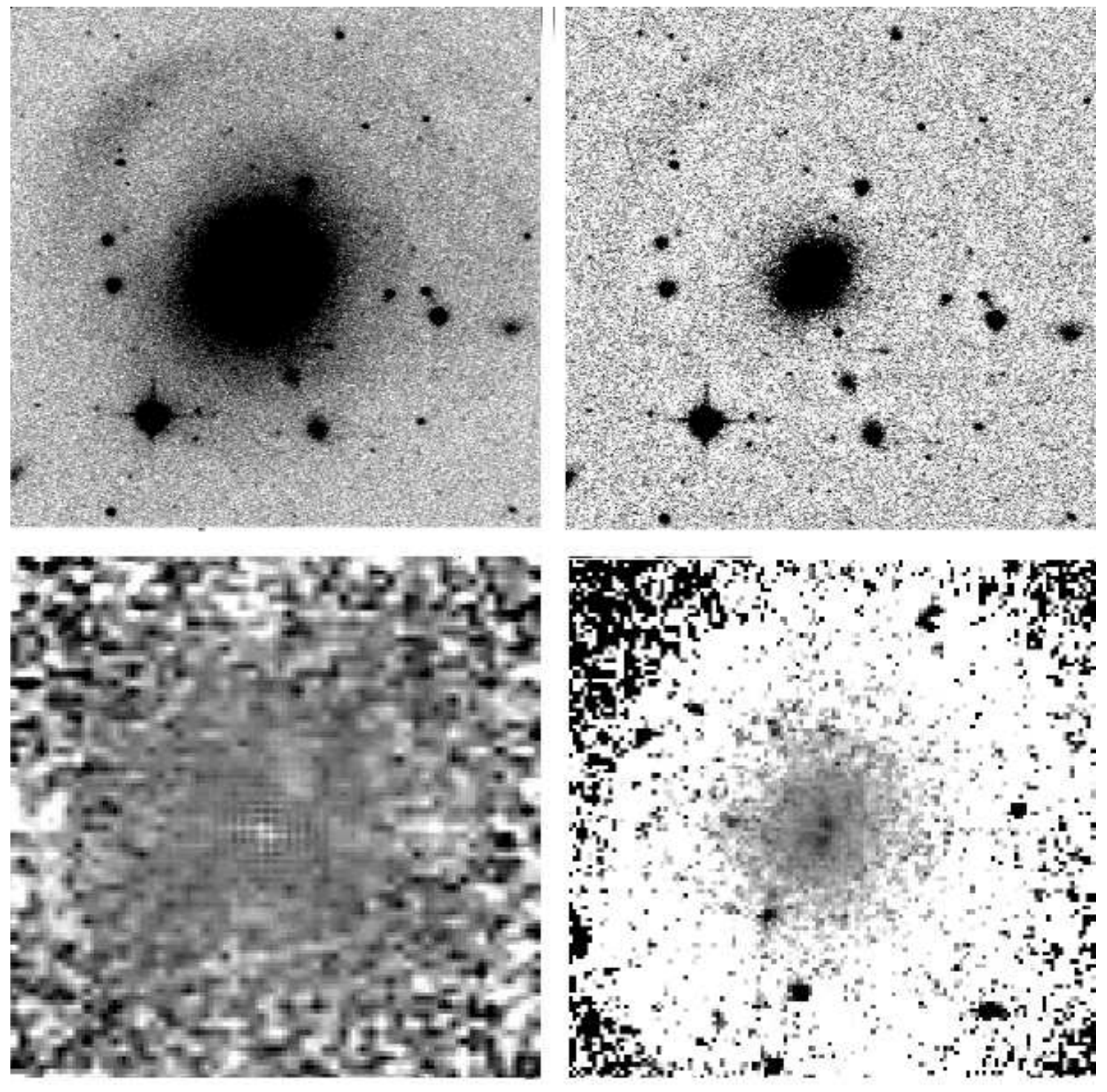

Fig. 4.- The analyzed images for NGC 3332. The plotted images correspond to: R-band (upper left), unsharp mask (upper right), R-band model division (lower left) and $\mathrm{R} / \mathrm{K}_{\mathrm{S}}$ (lower right), with scales on the sky of $3.5^{\prime} \times 3.5^{\prime}, 3.5^{\prime} \times 3.5^{\prime}, 90^{\prime \prime} \times 90^{\prime \prime}$, and $90^{\prime \prime} \times 90^{\prime \prime}$ respectively. Notice the shell/tidal features visible to the northeast of the galaxy in the unsharp masked image. The Rmodel image has a very weak quadrupole feature. Large symmetrical dust features are present in the color map image. For the model division and color maps, darker pixels indicate redder regions. 

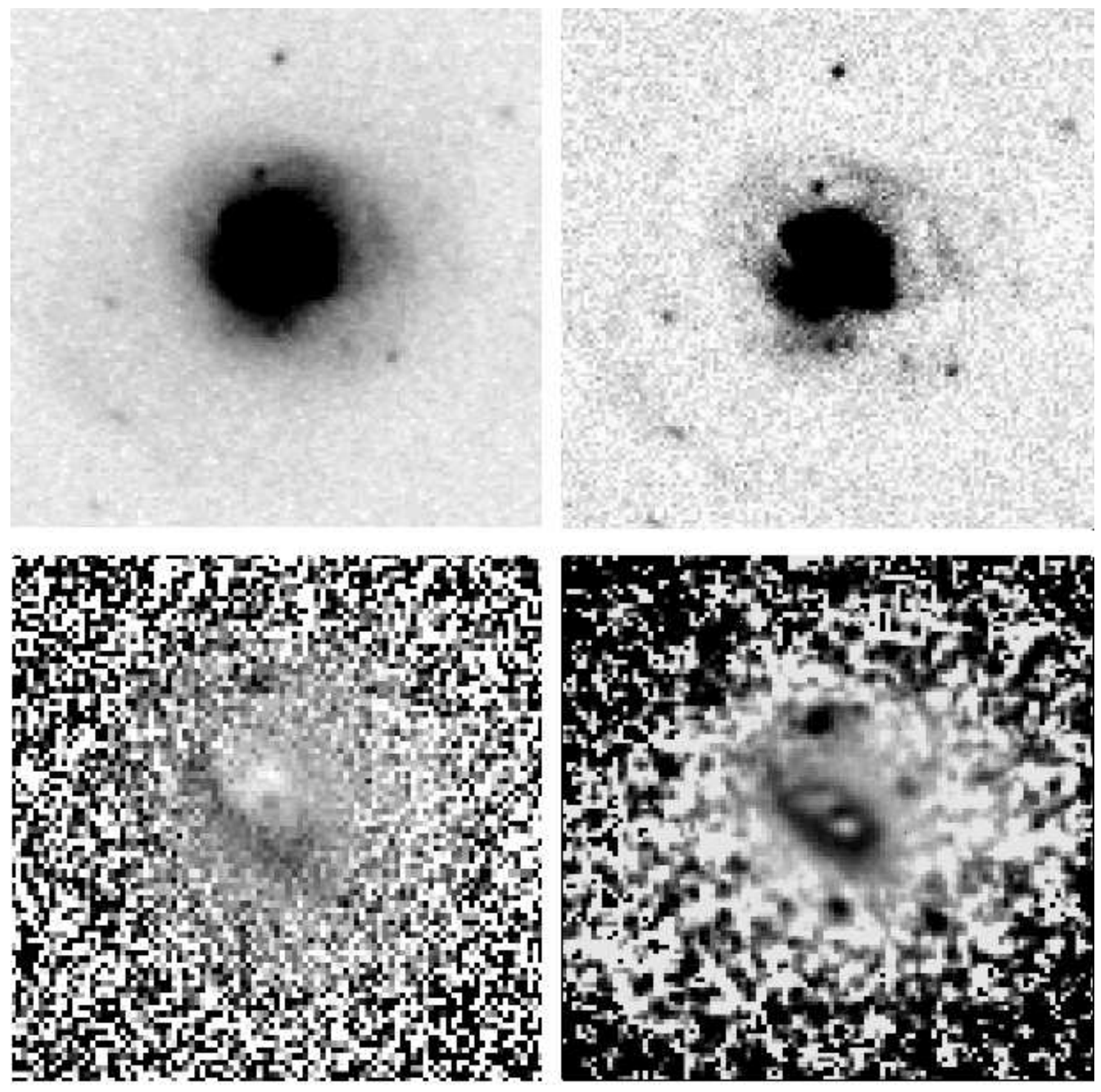

Fig. 5.- The analyzed images for IC 2637. The plotted images correspond to: R-band (upper left), unsharp mask (upper right), $\mathrm{B} / \mathrm{R}$ (lower left) and $\mathrm{R} / \mathrm{K}_{\mathrm{S}}$ (lower right), with scales on the sky of $90^{\prime \prime} \times 90^{\prime \prime}, 90^{\prime \prime} \times 90^{\prime \prime}, 60^{\prime \prime} \times 60^{\prime \prime}$, and $60^{\prime \prime} \times 60^{\prime \prime}$ respectively. Note the prominent tidal features and extensive dust structures. For the color maps, darker pixels indicate redder regions. 

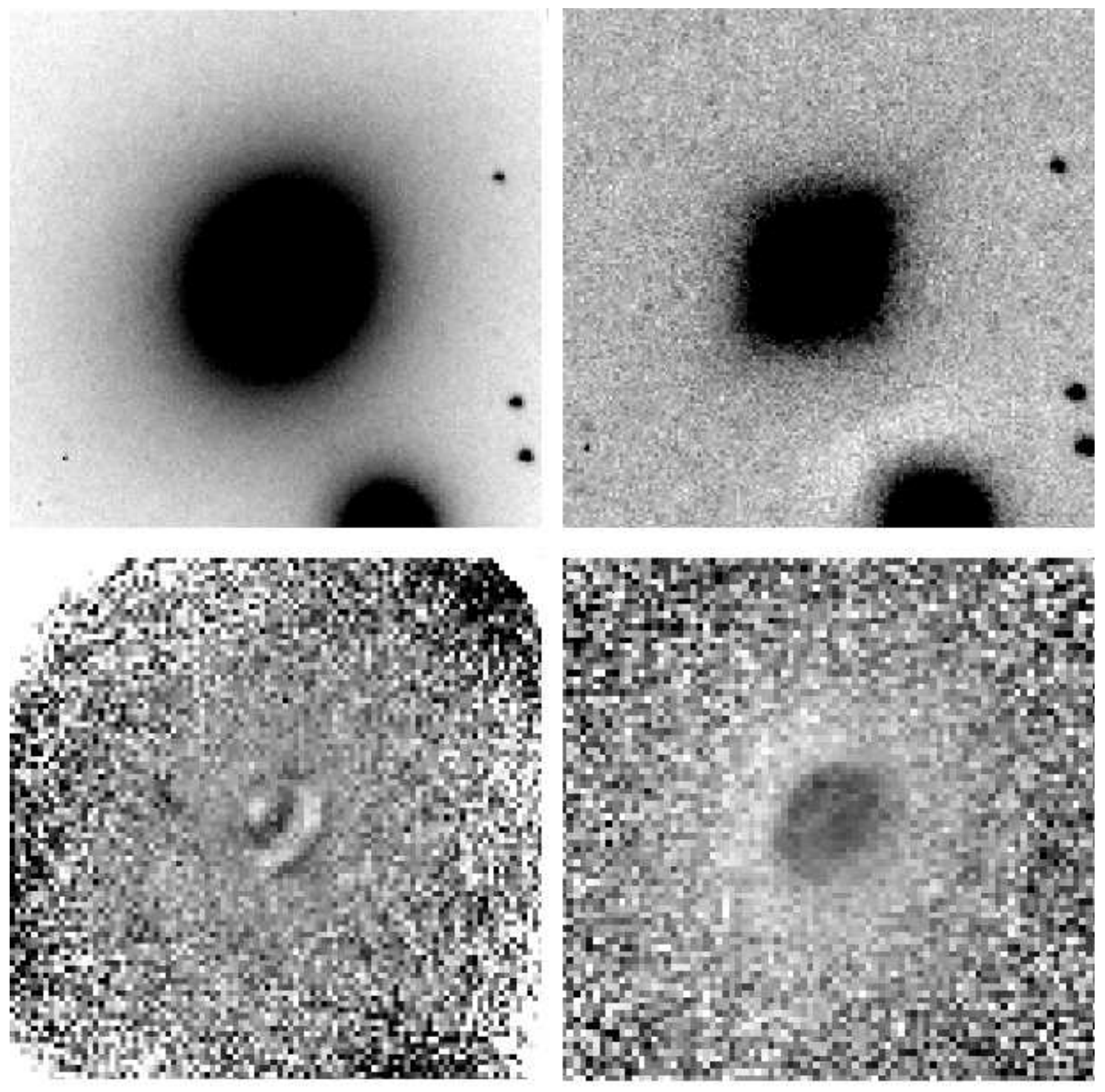

Fig. 6.- The analyzed images for NGC 383. The plotted images correspond to: I-band (upper left), unsharp mask (upper right), I-band model division (lower left) and B/I (lower right), with scales on the sky of $120^{\prime \prime} \times 120^{\prime \prime}, 120^{\prime \prime} \times 120^{\prime \prime}, 60^{\prime \prime} \times 60^{\prime \prime}$, and $60^{\prime \prime} \times 60^{\prime \prime}$ respectively. While the unsharp mask reveals no features, prominent dust structure is visible in both the model division image and color map. For the model division and color maps, darker pixels indicate redder regions. 

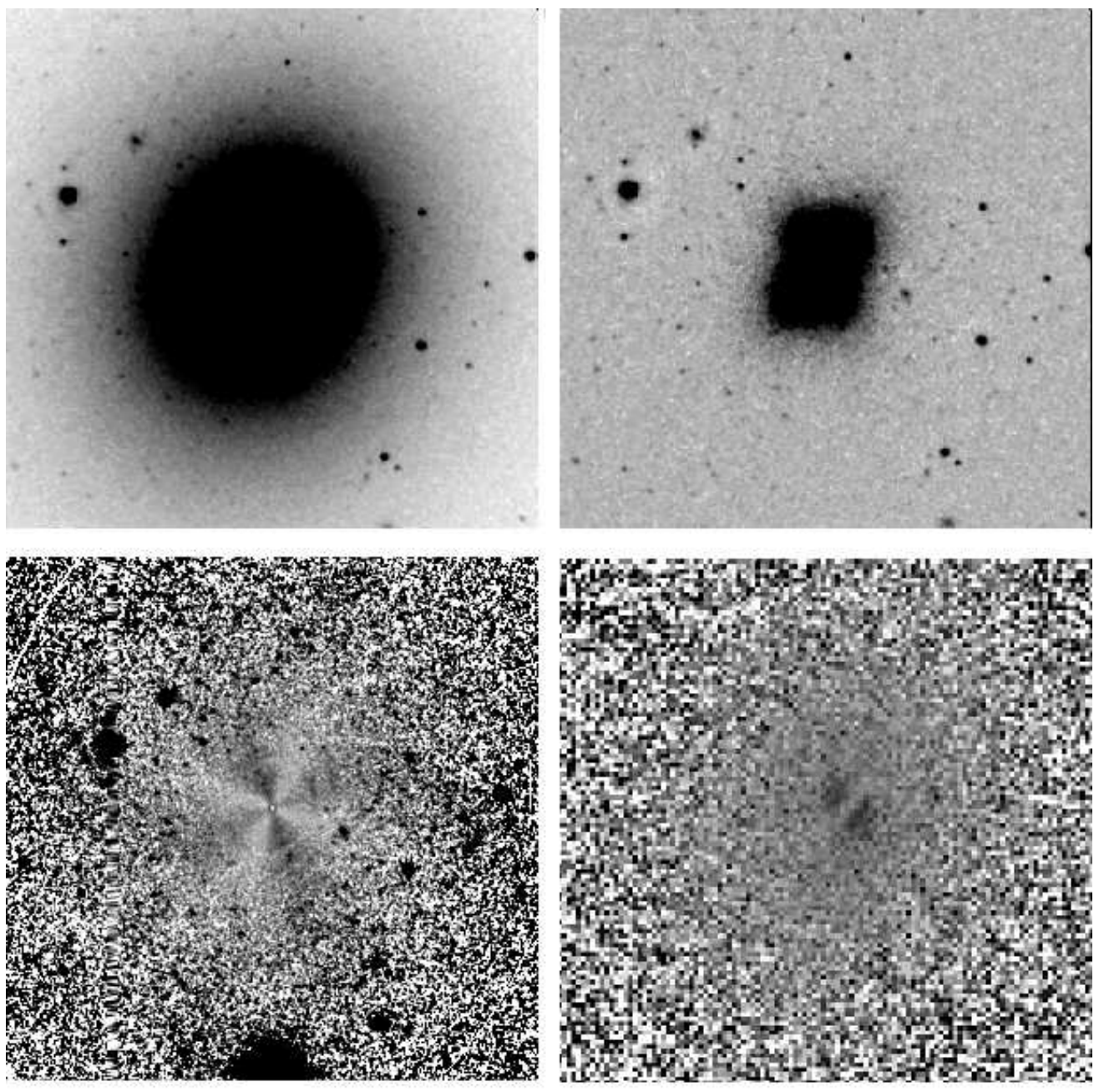

Fig. 7.- The analyzed images for NGC 4261. The plotted images correspond to: R-band (upper left), unsharp mask (upper right), R-band model division (lower left) and B/R (lower right), with scales on the sky of $3^{\prime} \times 3^{\prime}, 3^{\prime} \times 3^{\prime}, 3.5^{\prime} \times 3.5^{\prime}$, and $90^{\prime \prime} \times 90^{\prime \prime}$ respectively. No features are seen in the unsharp mask image. NGC 4261 is clearly boxy, which is the likely explanation for the quadrupole observed in the model-division image (Lauer 1985). Note how the phase of the quadrupole aligns roughly with the diagonal axis of the galaxy's box in the unsharp mask image. With higher resolution images, several authors have detected a central dust disk in this galaxy (Kormendy \& Stauffer 1986; Jaffe et al. 1996). The B/R color map also reveals complex structure in the core. For the model division and color maps, darker pixels indicate redder regions. 
Table 1. Isolated Early-Type Sample

\begin{tabular}{|c|c|c|c|c|c|}
\hline Galaxy & $\alpha(2000)$ & $\delta(2000)$ & $\mathrm{cz}$ & Type & Comments \\
\hline NGC 179 & 003746.2 & -175057 & 6027 & -3.0 & $\cdots$ \\
\hline NGC 766 & 015842.0 & 082048 & 8104 & -5.0 & $\cdots$ \\
\hline NGC 1132 & 025251.8 & -011628 & 6953 & -4.5 & $\begin{array}{l}\text { NGC } 11268.4^{\prime} \text { away; } \\
\text { group-like X-ray halo }\end{array}$ \\
\hline $\mathrm{A} 0300+16$ & 030315.0 & 162619 & 9740 & -5.0 & $\cdots$ \\
\hline UGC 2748 & 032754.2 & 023342 & 9060 & -4.7 & poor group \\
\hline $\mathrm{A} 0356+10$ & 035854.4 & 102602 & 9130 & -5.0 & low Galactic latitude \\
\hline IIZw017 & 043513.8 & -014353 & 9730 & -5.0 & MCG +00-12-049 21.8' away \\
\hline NGC 2110 & 055211.4 & -072722 & 2284 & -3.0 & $\begin{array}{r}\text { Seyfert } 2 \text { galaxy; } \\
\text { low Galactic latitude }\end{array}$ \\
\hline A0718-34 & 072047.5 & -340707 & 8900 & -3.0 & low Galactic latitude \\
\hline NGC 3209 & 102038.5 & 253015 & 6107 & -5.0 & poor group ${ }^{b}$ \\
\hline NGC 3332 & 104028.6 & 091059 & 5727 & -3.0 & $\cdots$ \\
\hline IC 2637 & 111349.8 & 093511 & 8763 & -4.0 & $\begin{array}{r}\text { Seyfert } 1.5 \text { galaxy; } \\
\text { FGC } 148 \text { A } 7.0^{\prime} \text { away }\end{array}$ \\
\hline IC 2980 & 115730.0 & -734106 & 8352 & -5.0 & low Galactic latitude \\
\hline ESO 505-G015 & 120707.9 & -254134 & 7467 & -4.0 & ESO 505-G017 11.8' away \\
\hline ESO 065-G001 & 123712.0 & -723531 & 7058 & -5.0 & $\begin{array}{r}\text { WKK } 1219 \text { 11.0' away; } \\
\text { low Galactic latitude }\end{array}$ \\
\hline ESO 574-G017 & 124035.0 & -203345 & 8583 & -4.0 & $\cdots$ \\
\hline NGC 5413 & 135753.5 & 645440 & 9634 & -5.0 & $\cdots$ \\
\hline UGC 9874 & 152714.9 & 770925 & 5380 & -5.0 & $\cdots$ \\
\hline UGC 10115 & 155707.8 & 635503 & 9216 & -5.0 & MCG +11-19-030 4.6' away \\
\hline IC 1156 & 160037.3 & 194324 & 9475 & -5.0 & $\ldots$ \\
\hline NGC 6172 & 162210.3 & -013054 & 4979 & -4.0 & $\cdots$ \\
\hline $\mathrm{A} 1836+17$ & 183825.4 & 171151 & 5090 & -3.0 & UGC $1130121.4^{\prime}$ away \\
\hline NGC 6702 & 184657.6 & 454220 & 4727 & -5.0 & $\cdots$ \\
\hline NGC 6799 & 193216.1 & -555429 & 5590 & -3.7 & $\cdots$ \\
\hline NGC 6849 & 200616.2 & -401151 & 6040 & -3.0 & $\cdots$ \\
\hline NGC 6944 & 203823.8 & 065947 & 4417 & -3.0 & NGC 6944A $6.3^{\prime}$ away \\
\hline NGC 7010 & 210439.5 & -122018 & 8486 & -4.0 & $\ldots$ \\
\hline IC 1392 & 213532.6 & 352355 & 4395 & -3.0 & $\begin{array}{l}\text { UGC } 117754.2^{\prime} \text { away; } \\
\text { UGC } 1178122.4^{\prime} \text { away; }\end{array}$ \\
\hline
\end{tabular}


Table 1-Continued

\begin{tabular}{rcccccc}
\hline \hline Galaxy & $\alpha(2000)$ & $\delta(2000)$ & cz & Type & Comments \\
\hline & & & & & low Galactic latitude \\
IC 5258 & 225131.6 & 230450 & 7747 & -3.0 & $\ldots$ \\
NGC 7618 & 231947.3 & 425108 & 5189 & -5.0 & UGC 12491 14.1' away; \\
& & & & & low Galactic latitude \\
\hline
\end{tabular}

References. - (a) Mulchaey \& Zabludoff (1999); (b) Ramella et al. (1997)

Table 2. Group Early-Type Sample

\begin{tabular}{rcrcccc}
\hline \hline Galaxy & $\alpha(2000)$ & $\delta(2000)$ & cz & Type & Comments \\
\hline NGC 383 & 010724.9 & 322445 & 5090 & -3.0 & $\ldots$ \\
NGC 533 & 012531.3 & 014533 & 5544 & -5.0 & $\ldots$ \\
NGC 2563 & 082035.7 & 210404 & 4480 & -2.0 & $\ldots$ \\
NGC 3091 & 100014.3 & -193813 & 3964 & -5.0 & $\ldots$ \\
NGC 3557 & 110957.4 & -373217 & 3067 & -5.0 & $\ldots$ \\
NGC 4261 & 121923.2 & 054931 & 2210 & -5.0 & $\ldots$ \\
NGC 4325 & 123306.7 & 103716 & 7709 & -5.0 & $\ldots$ \\
NGC 4759 & 125304.6 & -091202 & 3561 & -2.0 & member of HCG 62; \\
& & & & & & paired with NGC 4761 \\
NGC 4761 & 125305.8 & -091216 & 4259 & -1.0 & member of HCG 62; \\
& & & & & paired with NGC 4759 \\
NGC 5044 & 131524.0 & -162306 & 2704 & -5.0 & $\ldots$ \\
NGC 5129 & 132410.0 & 135836 & 6908 & -5.0 & $\ldots$ \\
IC 4296 & 133639.4 & -335800 & 3761 & -5.0 & $\ldots$ \\
NGC 5846 & 150629.2 & 013621 & 1822 & -5.0 & $\ldots$ \\
\hline
\end{tabular}


Table 3. Properties of Isolated Early-type Galaxies

\begin{tabular}{|c|c|c|c|c|c|c|c|}
\hline Galaxy & Telescope $^{\mathrm{a}}$ & Bands & $\begin{array}{c}\mathrm{R} \\
\text { Mag }\end{array}$ & $\begin{array}{c}\text { B } \\
\text { Mag }\end{array}$ & $\begin{array}{l}\text { Unsharp } \\
\text { Masking }\end{array}$ & $\begin{array}{c}\text { Model } \\
\text { Division }\end{array}$ & $\begin{array}{l}\text { Color } \\
\text { Map }\end{array}$ \\
\hline NGC 179 & L40 & $\mathrm{R}, \mathrm{B}$ & 13.20 & 14.63 & no & no & dust \\
\hline NGC 766 & $\mathrm{~L} 40$ & $\mathrm{R}, \mathrm{B}$ & 12.58 & 14.07 & no & no & no \\
\hline NGC 1132 & $\mathrm{~L} 40$ & $\mathrm{R}, \mathrm{B}$ & 11.97 & 13.49 & no & no & no \\
\hline $\mathrm{A} 0300+16$ & L40 & $\mathrm{R}, \mathrm{B}$ & 14.46 & 15.96 & no & no & no \\
\hline UGC 2748 & L40 & $\mathrm{R}, \mathrm{B}$ & 13.53 & 15.19 & no & no & dust \\
\hline $\mathrm{A} 0356+10$ & L40 & $\mathrm{R}, \mathrm{B}$ & 14.20 & 15.77 & no & no & no \\
\hline IIZw017 & L40 & $\mathrm{R}, \mathrm{B}$ & 14.17 & 15.79 & no & dust & dust \\
\hline NGC 2110 & L40 & $\mathrm{R}, \mathrm{B}$ & 11.36 & 13.39 & no & dust & dust \\
\hline A0718-34 & L40 & $\mathrm{R}, \mathrm{B}$ & 13.32 & 15.35 & shells & dust & dust \\
\hline NGC 3209 & L40 & $\mathrm{R}, \mathrm{B}$ & 11.68 & 13.21 & no & no & no \\
\hline NGC 3332 & L40,L100 & $\mathrm{K}_{\mathrm{S}}, \mathrm{R}, \mathrm{B}$ & 11.88 & 13.40 & shells & Quadrupole & dust \\
\hline IC 2637 & L40,L100 & $\mathrm{K}_{\mathrm{S}}, \mathrm{R}, \mathrm{B}$ & 12.72 & 14.07 & shells & $\ldots$ & dust \\
\hline IC 2980 & L40,L100 & $\mathrm{K}_{\mathrm{S}}, \mathrm{R}, \mathrm{B}$ & 11.58 & 13.47 & no & dust & dust \\
\hline ESO 505-G015 & L40 & $\mathrm{R}, \mathrm{B}$ & 12.51 & 14.26 & shells & no & dust \\
\hline ESO 065-G001 & L40,L100 & $\mathrm{K}_{\mathrm{S}}, \mathrm{R}, \mathrm{B}$ & 12.80 & 14.89 & shells & dust & dust \\
\hline ESO 574-G017 & L40,L100 & $\mathrm{K}_{\mathrm{S}}, \mathrm{R}, \mathrm{B}$ & 13.47 & 14.87 & shells & dust & dust \\
\hline IC 1156 & L100 & $\mathrm{K}_{\mathrm{S}}$ & $\ldots$ & $\ldots$ & $\ldots$ & $\ldots$ & $\ldots$ \\
\hline NGC 6172 & L40,L100 & $\mathrm{K}_{\mathrm{S}}, \mathrm{R}, \mathrm{B}$ & 12.61 & 13.98 & shells & dust & dust \\
\hline NGC 6799 & L40,L100 & $\mathrm{K}_{\mathrm{S}}, \mathrm{R}, \mathrm{B}$ & 12.01 & 13.51 & no & dust & dust \\
\hline NGC 7010 & L40,L100 & $\mathrm{K}_{\mathrm{S}}, \mathrm{R}, \mathrm{B}$ & 12.37 & 14.16 & shells & dust & dust \\
\hline IC 1392 & P60 & I,B & $\ldots$ & $\ldots$ & shells & no & dust \\
\hline IC 5258 & $\mathrm{P} 60$ & $\mathrm{I}, \mathrm{B}$ & $\cdots$ & $\cdots$ & no & no & dust \\
\hline NGC 7618 & P60 & $\mathrm{I}, \mathrm{B}$ & $\ldots$ & $\cdots$ & no & dust & dust \\
\hline
\end{tabular}

${ }^{\mathrm{a}} \mathrm{L} 40=$ Las Campanas 40-inch; L100= Las Campanas 100-inch; P60= Palomar 60-inch. 
Table 4. Properties of Early-type Group Galaxies

\begin{tabular}{rcrrrrrr}
\hline \hline Galaxy & Telescope $^{\mathrm{a}}$ & Bands & $\begin{array}{c}\mathrm{R} \\
\text { Mag }\end{array}$ & $\begin{array}{c}\text { B } \\
\text { Mag }\end{array}$ & $\begin{array}{r}\text { Unsharp } \\
\text { Masking }\end{array}$ & $\begin{array}{c}\text { Model } \\
\text { Division }\end{array}$ & $\begin{array}{r}\text { Color } \\
\text { Map }\end{array}$ \\
\hline NGC 383 & P60 & I,B & $\ldots$ & $\ldots$ & no & dust & dust \\
NGC 533 & L40 & R,B & b & b & no & no & no \\
NGC 2563 & L40 & R,B & 11.73 & 13.19 & no & no & dust \\
NGC 3091 & L40 & R,B & 10.59 & 12.24 & no & no & no \\
NGC 3557 & L40 & R,B & 9.89 & 11.43 & no & dust & dust \\
NGC 4261 & L40 & R,B & 9.73 & 11.27 & no & Quadrupole & dust \\
NGC 4325 & L40,L100 & K, R,B & 12.61 & 14.15 & no & no & dust \\
NGC 4759 & L40 & R,B & 12.50 & 13.97 & no & dust & dust \\
NGC 4761 & L40 & R,B & 11.46 & 13.00 & no & no & no \\
NGC 5044 & L40 & R,B & 10.02 & 11.61 & no & dust & dust \\
NGC 5129 & L40,L100 & K, R,B & 11.59 & 13.01 & shells & Quadrupole & dust \\
IC 4296 & L40 & R,B & 9.96 & 11.71 & no & dust & dust \\
NGC 5846 & L100 & KS & $\ldots$ & $\ldots$ & $\ldots$ & $\ldots$ & $\ldots$ \\
\hline
\end{tabular}

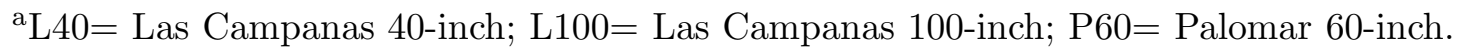

${ }^{\mathrm{b}}$ Data taken under non-photometric conditions, so no magnitudes are given. 